\title{
Automated volumetric modulated arc therapy planning for whole pelvic prostate radiotherapy
}

\author{
Martin Buschmann ${ }^{1,2} \cdot$ Abdul Wahab M. Sharfo $^{3} \cdot$ Joan Penninkhof $^{3}$. Yvette Seppenwoolde ${ }^{1,2}$. \\ Gregor Goldner ${ }^{1}$. Dietmar Georg ${ }^{1,2} \cdot$ Sebastiaan Breedveld ${ }^{3}$ Ben J. M. Heijmen ${ }^{3}$
}

Received: 16 July 2017 / Accepted: 25 November 2017 / Published online: 21 December 2017

(c) The Author(s) 2017. This article is an open access publication.

\begin{abstract}
Background For several tumor entities, automated treatment planning has improved plan quality and planning efficiency, and may enable adaptive treatment approaches. Whole-pelvic prostate radiotherapy (WPRT) involves large concave target volumes, which present a challenge for volumetric arc therapy (VMAT) optimization. This study evaluates automated VMAT planning for WPRT-VMAT and compares the results with manual expert planning.

Methods A system for fully automated multi-criterial plan generation was configured for each step of sequential-boost WPRT-VMAT, with final "autoVMAT" plans being automatically calculated by the Monaco treatment planning system (TPS; Elekta AB, Stockholm, Sweden). Configuration was based on manually generated VMAT plans (manualVMAT) of 5 test patients, the planning protocol, and discussions with the treating physician on wishes for plan improvements. AutoVMAT plans were then generated for another 30 evaluation patients and compared to manualVMAT plans. For all 35 patients, manualVMAT plans were optimized by expert planners using the Monaco TPS.

Results AutoVMAT plans exhibited strongly improved organ sparing and higher conformity compared to manualVMAT. On average, mean doses $\left(\mathrm{D}_{\text {mean }}\right)$ of bladder and rectum were reduced by 10.7 and $4.5 \mathrm{~Gy}$, respectively, by autoVMAT. Prostate target coverage $\left(\mathrm{V}_{95 \%}\right)$ was slightly higher $(+0.6 \%)$ with manualVMAT. In a blinded scoring session, the radiation oncologist preferred autoVMAT plans to manualVMAT plans for 27/30 patients. All treatment plans were considered clinically acceptable. The workload per patient was reduced by $>70 \mathrm{~min}$.

Conclusion Automated VMAT planning for complex WPRT dose distributions is feasible and creates treatment plans that are generally dosimetrically superior to manually optimized plans.
\end{abstract}

Keywords Prostate cancer $\cdot$ Lymph nodes $\cdot$ Volumetric modulated arc therapy $\cdot$ Organs at risk $\cdot$ Multicriteria optimization

Electronic supplementary material The online version of this article (https://doi.org/10.1007/s00066-017-1246-2) contains supplementary material, which is available to authorized users.

\footnotetext{
Martin Buschmann

martin.buschmann@akhwien.at

1 Department of Radiation Oncology, Medical University of Vienna/AKH Wien, Währinger Gürtel 18-20, 1090 Vienna, Austria

2 Christian Doppler Laboratory for Medical Radiation Research for Radiation Oncology, Medical University of Vienna, Vienna, Austria

3 Department of Radiation Oncology, Erasmus MC Cancer Institute, Rotterdam, The Netherlands
} 


\section{Automatisierte volumenmodulierte Arc-Therapieplanung für Ganzbecken-Prostatabestrahlung}

\section{Zusammenfassung}

Hintergrund Automatisierte Bestrahlungsplanung zeigte bei einigen Tumorentitäten Vorteile durch verbesserte Planqualität und höhere Planungseffizienz, was die Einführung adaptiver Bestrahlungstechniken erleichtern könnte. Die Ganzbecken-Prostatabestrahlung (WPRT) beinhaltet große konkave Zielvolumina und stellt eine Herausforderung für die Optimierung der volumenmodulierten Arc-Therapie (VMAT) dar. Die Studie evaluiert die automatisierte VMAT-Planung für WPRT und vergleicht die Ergebnisse mit der manuellen Planung.

Methoden Ein System für die vollautomatisierte multikriterielle Planung wurde für die WPRT mit sequenziellem Boost konfiguriert, wobei die finalen AutoVMAT-Pläne im Monaco-Planungssystem (Elekta AB, Stockholm, Schweden) berechnet wurden. Die Konfiguration basierte auf manuell erstellten VMAT-Plänen (ManualVMAT) von 5 Testpatienten, dem klinischen Planungsprotokoll und Diskussionen mit dem behandelten Arzt zu Planverbesserungen. AutoVMAT-Pläne wurden für weitere 30 Evaluierungspatienten erstellt und mit ManualVMAT-Plänen verglichen. Die ManualVMAT-Pläne wurden für alle 35 Patienten von erfahrenen Planern in Monaco optimiert.

Ergebnisse AutoVMAT-Pläne wiesen stark verbesserte Organschonung und höhere Konformität im Vergleich zu ManualVMAT-Plänen auf. Die mittlere Dosis $\left(D_{\text {mean }}\right.$ ) von Harnblase und Rektum wurden im Durchschnitt um 10,7 und 4,5 Gy mit AutoVMAT reduziert. Die Zielgebietsabdeckung der Prostata $\left(\mathrm{V}_{95 \%)}\right.$ war für ManualVMAT etwas höher $(+0,6 \%)$. In einer Blindbewertung bevorzugte der behandelnde Radioonkologe den AutoVMAT-Plan gegenüber dem ManualVMATPlan bei 27/30 Patienten. Alle Bestrahlungspläne wurden als klinisch akzeptabel bewertet. Der manuelle Arbeitsaufwand pro Patient reduzierte sich um $>70 \mathrm{~min}$.

Schlussfolgerung Die automatisierte VMAT-Planung ist für komplexe Dosisverteilungen bei WPRT durchführbar und generiert im Allgemeinen Bestrahlungspläne, die den manuell optimierten Plänen dosimetrisch überlegen sind.

Schlüsselwörter Prostatakrebs $\cdot$ Lymphknoten $\cdot$ Volumenmodulierte Arc-Therapie $\cdot$ Risikoorgane $\cdot$ Multikriterielle Optimierung

Modern linear accelerator (linac)-based prostate radiotherapy (RT) is commonly delivered by intensity-modulated radiation therapy (IMRT) and, more recently, by volumetric modulated arc therapy (VMAT). However, treatment planning is often an iterative trial-and-error process, and the resulting plan quality is strongly dependent on the planner's experience.

The clinical target volume (CTV) usually comprises the prostate gland, but in intermediate- and high-risk disease, the seminal vesicles and pelvic lymph nodes are also commonly irradiated. This technique of whole-pelvic prostate RT (WPRT) has shown clinical benefit compared to local prostate-only RT in several studies [1, 2], but its role remains controversial $[3,4]$. The optimization of WPRTIMRT/VMAT plans is more challenging and time consuming than for the local prostate treatments, due to the larger and more complex target volumes.

In recent years, multi-criterial optimization (MCO) has been applied in the field of treatment plan optimization with the aim of generating Pareto-optimal plans which cannot be improved further in one aspect without worsening another. Another motivation for advanced optimization is avoidance of time-consuming iterative treatment planning, which may still lead to treatments lacking Pareto optimality. An MCO approach developed by Craft et al. [5, 6] uses manual navigation of a plan library that spans the Pareto space. This strategy can be classified as an a posteriori technique, where manual interaction by the user is necessary to select the best-fitting treatment plan. Erasmus-iCycle is a multi-criterial optimizer for beam profiles and beam angle selection [7] which employs an a priori strategy. The user defines a treatment site-specific optimization protocol, a so-called wishlist, containing the goal functions that are optimized in a specific order, defined by assigned priorities. The priorities steer the fully automated multi-criterial plan generation. In addition, the wishlist can contain hard constraints that must not be violated. Generated plans are Pareto optimal and clinically favorable [8-10]. Currently, Erasmus-iCycle is used as a pre-optimizer; optimized iCycle plans are subsequently reconstructed with Monaco treatment planning system (TPS; Elekta AB, Stockholm, Sweden) to generate deliverable IMRT or VMAT plans [9, 11]. After the development of a wishlist and a translation strategy to the TPS in a multidisciplinary team, treatment planning with Erasmus-iCycle/Monaco is fully automatic. The algorithm is successfully applied in clinical routine for several tumor sites, including head and neck [8], cervix [12], lung [10], spinal metastases [13], and local prostate cancer [9]. Up until now, the algorithm has only been validated against manual planning at a single institution, and the previously demonstrated benefit of autoplanning may be explainable by institution-specific protocols. 
This study investigates the feasibility of automated generation of VMAT plans for WPRT with Erasmus-iCycle/ Monaco and compares the resulting dose distributions with manually optimized VMAT plans that were generated by expert planners in a different center, also using Monaco.

\section{Materials and methods}

\section{Patients}

From the clinical database of the department of Radiation Oncology in Vienna, 35 prostate cancer patients who were treated with WPRT-VMAT between May 2014 and July 2016 were randomly selected for this study. Patients with hip implants and bladder filling less than $100 \mathrm{ml}$ on the planning CT scan were not included. Each patient had gold fiducials implanted in the prostate for improved target localization during image guidance. A CT scan with $2 \mathrm{~mm}$ slice thickness was used for treatment planning. Patients were scanned and treated with a rectal balloon. The prostate was delineated as the primary CTV (CTV-P), and the pelvic lymph nodes including seminal vesicles as well as the CTV$\mathrm{P}$ were defined as the secondary CTV (CTV-LN). Rectum, bladder, bowel bag, and femoral heads were manually contoured as organs at risk (OAR). The bowel was delineated up to a $2 \mathrm{~cm}$ extension of the target in the cranial direction. Considering the use of daily image guidance, setup margins of $5 \mathrm{~mm}$ were applied to create two planning target volumes: PTV-P and PTV-LN.

The total radiation dose was prescribed in a two-phase Vienna-specific protocol: the first phase delivered 60 and

Table 1 Clinical dose constraints for organs at risk for the total summed dose

\begin{tabular}{lll}
\hline Rectum & $\mathrm{D}_{\max }$ & $78 \mathrm{~Gy}$ \\
& $\mathrm{~V}_{65 \mathrm{~Gy}}$ & $20 \%$ \\
& $\mathrm{~V}_{60 \mathrm{~Gy}}$ & $40 \%$ \\
& $\mathrm{~V}_{55 \mathrm{~Gy}}$ & $45 \%$ \\
& $\mathrm{~V}_{50 \mathrm{~Gy}}$ & $50 \%$ \\
Bowel & $\mathrm{D}_{\max }$ & $56 \mathrm{~Gy}$ \\
bag & $\mathrm{V}_{50 \mathrm{~Gy}}$ & $10 \%$ \\
& $\mathrm{~V}_{45 \mathrm{~Gy}}$ & $15 \%$ \\
& $\mathrm{~V}_{40 \mathrm{~Gy}}$ & $20 \%$ \\
Bladder & $\mathrm{D}_{\text {max }}$ & $78 \mathrm{~Gy}$ \\
& $\mathrm{~V}_{65 \mathrm{~Gy}}$ & $20 \%$ \\
& $\mathrm{~V}_{55 \mathrm{~Gy}}$ & $40 \%$ \\
& $\mathrm{~V}_{50 \mathrm{~Gy}}$ & $50 \%$ \\
& $\mathrm{~V}_{35 \mathrm{~Gy}}$ & $80 \%$ \\
Femoral & $\mathrm{D}_{\text {max }}$ & $55 \mathrm{~Gy}$ \\
heads & $\mathrm{V}_{45 \mathrm{~Gy}}$ & $5 \%$ \\
\hline
\end{tabular}

$D_{\max }$ maximum dose, $V_{x G y}$ relative volume of organ receiving at least x Gy
50 Gy in 25 fractions to PTV-P and PTV-LN, respectively, using a simultaneous integrated boost (SIB) technique. The second phase was a sequential boost of $13 \mathrm{~Gy}$ in 5 fractions to PTV-P. Clinical acceptability of the treatment plans was assessed through a list of clinically applied organ dose constraints. which is presented in Table 1 , and by clinical judgment of the treating physician.

\section{Treatment planning and delivery systems}

Prior to initiation of this study, discussions between the radiation oncologist (GG), the dosimetrist, and the physicists (MB, AS, YS, JP, BH) were held to define the guidelines and preferences for dose distributions. A target dose criterion was defined as a minimum target coverage corresponding to total volume irradiated with $95 \%$ of the prescribed dose $\left(\mathrm{V}_{95 \%}\right)=96 \%$ for PTV-P and PTV-LN in the total summed dose (phase 1 plus phase 2). The physician expressed his preference for high target coverage and therefore the aim was to reach approximately $\mathrm{V}_{95 \%}=98 \%$ during optimization of the two single treatment plans.

All final autoVMAT and manualVMAT plans were generated with the Monaco TPS version 5.11 for a VersaHD linac (Elekta) with $10 \mathrm{MV}$ photons. All treatments were planned to be delivered by full $360^{\circ}$ coplanar arcs. Phase 1 was planned as dual arc and the boost phase 2 as single arc, with the isocenter being placed in the center of PTV-LN and PTV-P, respectively.

\section{Manual VMAT planning}

Two experienced planners from the Vienna team performed VMAT treatment planning (manualVMAT) in Monaco for all 35 patients according to current clinical guidelines, with commonly used templates of optimization cost functions. The employed templates are described in the supplementary material. For each patient, the optimization parameters were iteratively tweaked to improve the dose distributions. This process was performed in the absence of time constraints and the planner only stopped when no further improvement in plan quality could be achieved. The two plan phases were optimized and finalized independently before the summed dose was inspected to check the OAR constraints. Each patient was planned by just one planner.

\section{Automated VMAT planning with Erasmus-iCycle/ Monaco}

For automated VMAT planning (autoVMAT), the ErasmusiCycle algorithm [7] was used as a pre-optimizer. To this purpose, a VMAT plan was approached by using 20 equiangularly positioned IMRT beams. The two wishlists for the two treatment phases used in this study are presented in 
Table 2 Applied wishlists for automatic volumetric modulated arc therapy plan generation for (A) phase 1 (i. e., whole pelvis) and (B) phase 2 (i. e., boost) plans. A detailed description is included in the supplementary material. The parameters $\mathrm{k}$ and $\alpha$ are specific to the iCycle algorithm [7]

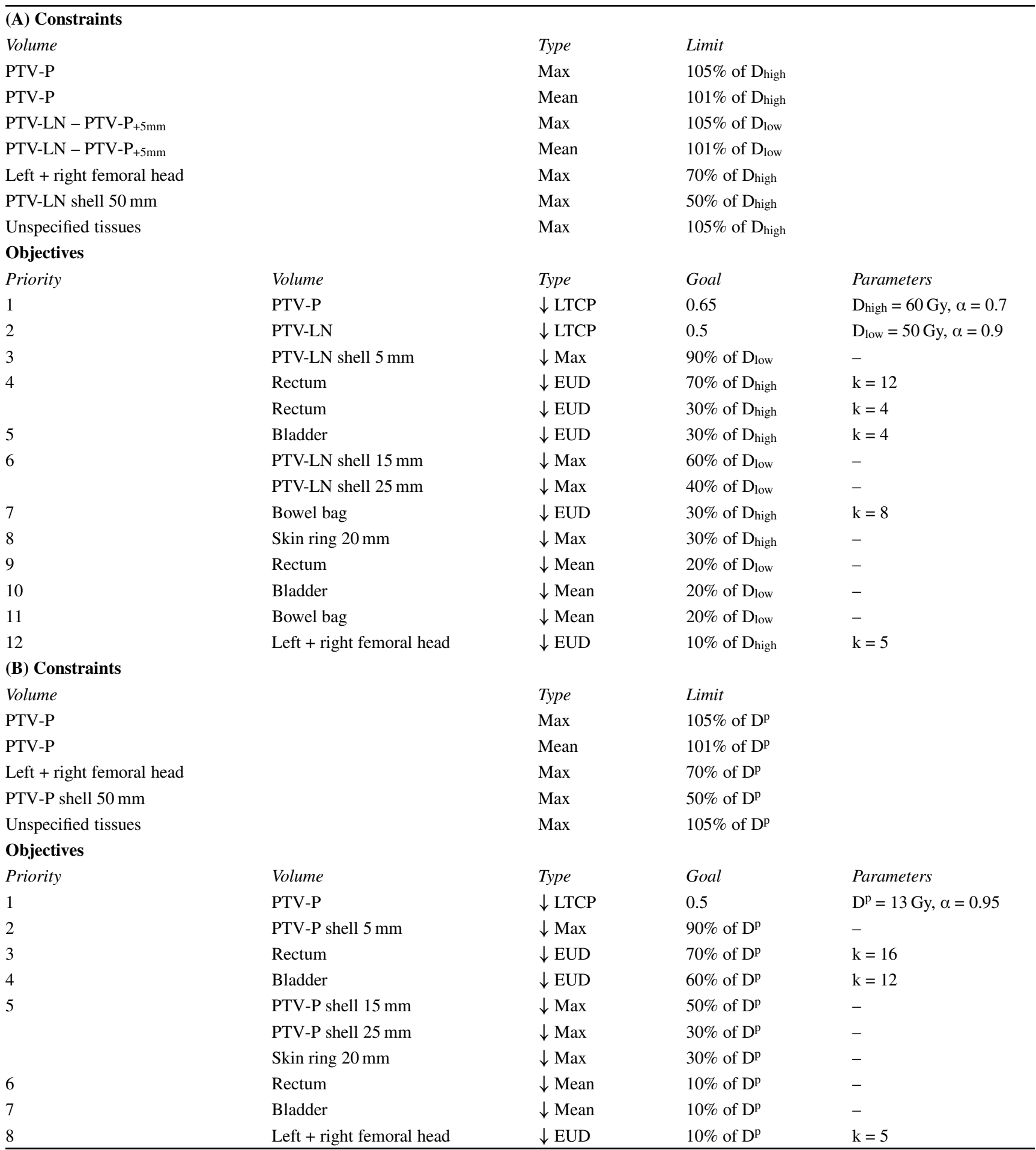

$P T V$-P planning target volume of the prostate, $D^{p}$ prescribed dose, $L T C P$ logarithmic tumor control probability, EUD equivalent uniform dose, $\alpha$ cell sensitivity, $k$ volume effect, $P T V-L N$ planning target volume of the lymph nodes, $D_{\text {high }}$ high (=60 Gy) prescribed dose, $D_{\text {low }}$ low (=50 Gy) prescribed dose, $P T V-P+5 m m$ a $5 \mathrm{~mm}$ transition region within $\mathrm{PTV}_{\mathrm{LN}}$ 
Table 2 and a detailed description is provided in the supplementary material. The segmentation settings in Monaco were identical for manual and automated plan generation, and are described in the supplementary material.

The autoVMAT strategy was developed using a random subset of 5 of the total of 35 study patients. This training cohort was used to configure the Erasmus-iCycle/Monaco system for high-quality automated VMAT plan generation, i. e., development of the site-specific wishlists and automatic translations of pre-optimization results into patientspecific Monaco templates for both treatment phases. A first strategy for autoVMAT was created according to clinical guidelines and applied on the training cohort to create deliverable VMAT plans. A "flavor session" with the radiation oncologist and medical physicists was then held, where manualVMAT and autoVMAT plans for the training patients were blindly presented and discussed. Using the results and physicians' preferences from this flavor session, the autoVMAT configurations were refined and then applied to the 30 patients not used for configuration (evaluation patients).

\section{Dosimetric plan evaluations}

For the 30 evaluation patients, the dose distributions of the individual treatment phases and the summed total dose as generated by autoVMAT and manualVMAT were analyzed and compared. Normal tissue sparing was assessed by extracting the near-maximum dose $\mathrm{D}_{2 \%}$ and mean dose ( $\left.\mathrm{D}_{\text {mean }}\right)$ for each OAR, and the total volume irradiated at 95 and $50 \%$ of the respective prescription dose. Additionally, for the rectum, $\mathrm{V}_{70 \mathrm{~Gy}}$ and $\mathrm{V}_{55 \mathrm{~Gy}}$ were assessed in the summed dose, as these parameters are correlated to rectal toxicity [14] (adjusted for hypofractionation according to the linear quadratic model with $\alpha / \beta=3$ ). For target structures, $V_{95 \%}$ (target coverage), $\mathrm{D}_{2 \%}$, and $\mathrm{D}_{\text {mean }}$ were analyzed. The homogeneity $(\mathrm{HI})$ and two conformity indices $\left(\mathrm{CI}, \mathrm{CI}_{50 \%}\right)$ were calculated as follows:

$H I=\left(D_{2 \%}-D_{98 \%}\right) / D_{50 \%}$,

$C I=V_{95 \%} / V_{\mathrm{PTV}}$,

$C l_{50 \%}=V_{50 \%} / V_{\mathrm{PTV}}$,

where $\mathrm{V}_{95 \%}$ and $\mathrm{V}_{50 \%}$ describe the total volumes irradiated with 95 and $50 \%$ of the prescribed dose, respectively. For the determination of $\mathrm{D}_{2} \%, \mathrm{D}_{\text {mean }}$, and $\mathrm{HI}$ in the PTV-LN, the SIB volume including a $2 \mathrm{~cm}$ margin was subtracted.

Dosimetric differences between autoVMAT and manualVMAT were tested for statistical significance by a twosided Wilcoxon matched-paired signed-rank test $(p<0.05)$. No rescaling of dose distributions was performed, since $\mathrm{D}_{\text {mean }}$ was equivalent in the total plan sum for autoVMAT and manualVMAT. The number of monitor units (MU) for each of the two treatment phases was noted.

\section{Physician's plan scoring}

In a blinded plan scoring session, the radiation oncologist was simultaneously presented with the total summed plans created by autoVMAT and manualVMAT for all 30 study patients. The cumulative DVHs for all structures, the dose distributions in the TPS, and a table with the most important dosimetric parameters were provided to the physician. Based on this information, the physician rated the two distributions relatively to each other according to three categories:

- Plan A is considerably better than plan B

- Plan B is considerably better than plan A

- Plan A and plan B are of similar quality

\section{Dosimetric verification measurements}

Deliverability of autoVMAT and manualVMAT plans was checked by performing verification dose measurements for 10 randomly selected patients. Dose distributions were measured with the Delta ${ }^{4}$ phantom (ScandiDos, Uppsala, Sweden) and compared to planned doses through a gamma analysis (3\% local dose difference, $3 \mathrm{~mm}$ ).

\section{Results}

\section{Dosimetric plan evaluations}

All generated autoVMAT and manualVMAT dose distributions fulfilled the criteria for clinical acceptability in terms of OAR sparing and target coverage. Population average DVHs for the 30 evaluation patients for the single plan phases and the total treatment are shown in Fig. 1, pointing toward meaningful advantages for autoVMAT. Dose-volume parameters of autoVMAT and differences with manualVMAT are presented in Table 3.

Fig. 2 depicts an exemplary dose distribution in an axial slice of the summed treatment for both optimization techniques.

In general, dose distributions in the targets were similar, with small differences as is shown by the DVHs and Table 3. $\mathrm{D}_{\text {mean }}$ in PTV-P was identical in phase 1 and the summed dose in both optimizations. Target coverage was similar for both optimization methods, but was statistically significantly higher in manualVMAT in the PTV-P by a small amount of $0.6 \%$ points on average. In the PTV-LN, $\mathrm{V}_{95 \%}$ was higher in autoVMAT in the summed plan. Significantly lower OAR doses were observed in rectum, bladder, and 

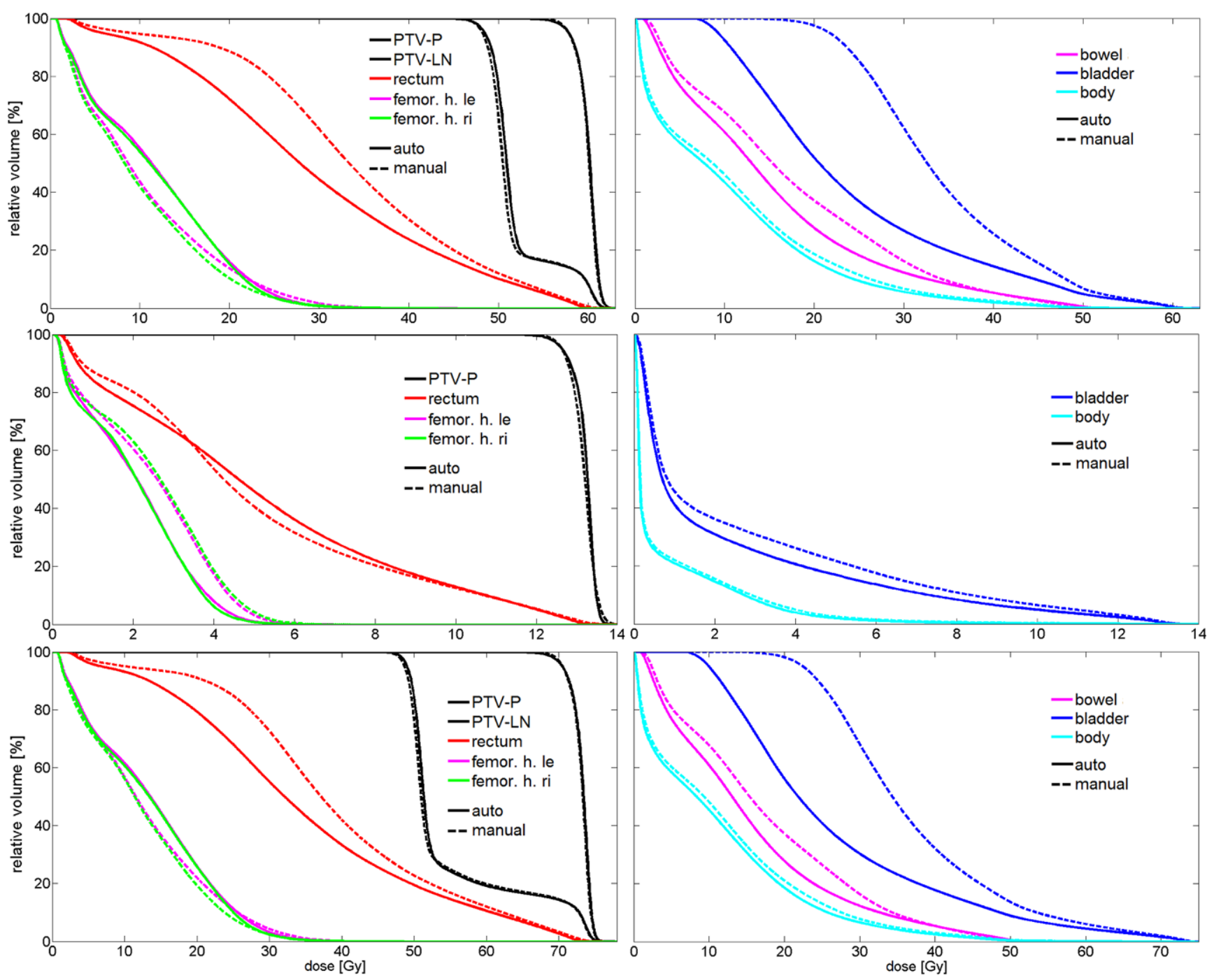

Fig. 1 Mean dose-volume histograms (DVHs) for automated and manual volumetric modulated arc therapy (autoVMAT, manualVMAT) plans for the 30 evaluation patients. Top: phase 1 whole-pelvis plan; middle: phase 2 boost plan; bottom: sum plan; femor. $h$. femoral head. PTV-P planning target volume prostate, $P T V-L N$ planning target volume lymph nodes

bowel in autoVMAT compared to manualVMAT, for both phases and for the sum plan. Most prominent was the lower bladder dose in autoVMAT, with average $\mathrm{D}_{\text {mean }}$ values in the sum plan of 26.3 and $37 \mathrm{~Gy}$ for autoVMAT and manualVMAT, respectively. Doses to the femoral heads were generally higher in autoVMAT, but the average difference in $\mathrm{D}_{\text {mean }}$ was below $1.7 \mathrm{~Gy}$.

AutoVMAT dose distributions were more conformal than manualVMAT doses, which was especially evident in the smaller $\mathrm{CI}_{50 \%}$ in both planning phases. The volume of the $50 \%$ isodose was decreased on average by $852 \mathrm{ml}$ in phase $1(50 \%=25 \mathrm{~Gy})$ and $63 \mathrm{ml}$ in phase $2(50 \%=$ $6.5 \mathrm{~Gy}$ ) with autoVMAT. ManualVMAT plans delivered slightly more homogeneous target doses. The average number of MUs were 664/1029 for phase 1 and 563/716 for phase 2 in manualVMAT and autoVMAT, respectively.

\section{Physician's plan scoring}

During the blinded scoring session, the radiation oncologist considered all plans from both optimizations to be clinically acceptable. He preferred the autoVMAT plan in 27 out of 30 patients and the manualVMAT in the other 3 patients. He chose the autoVMAT plans mainly for their increased OAR sparing, while the occasional small advantages of manualVMAT in PTV-P coverage were not considered clinically relevant in most cases. However, in the 3 patients for whom manualVMAT was considered better, the $\mathrm{V}_{95 \%}$ and $\mathrm{D}_{\min }$ were noticeably lower in autoVMAT. After the scoring session, the MUs of the treatment plans in these 3 patients were rescaled to reach identical values of $\mathrm{V}_{95 \%}$ in the PTV-P in the autoVMAT and manualVMAT plans, and another blinded scoring session was 


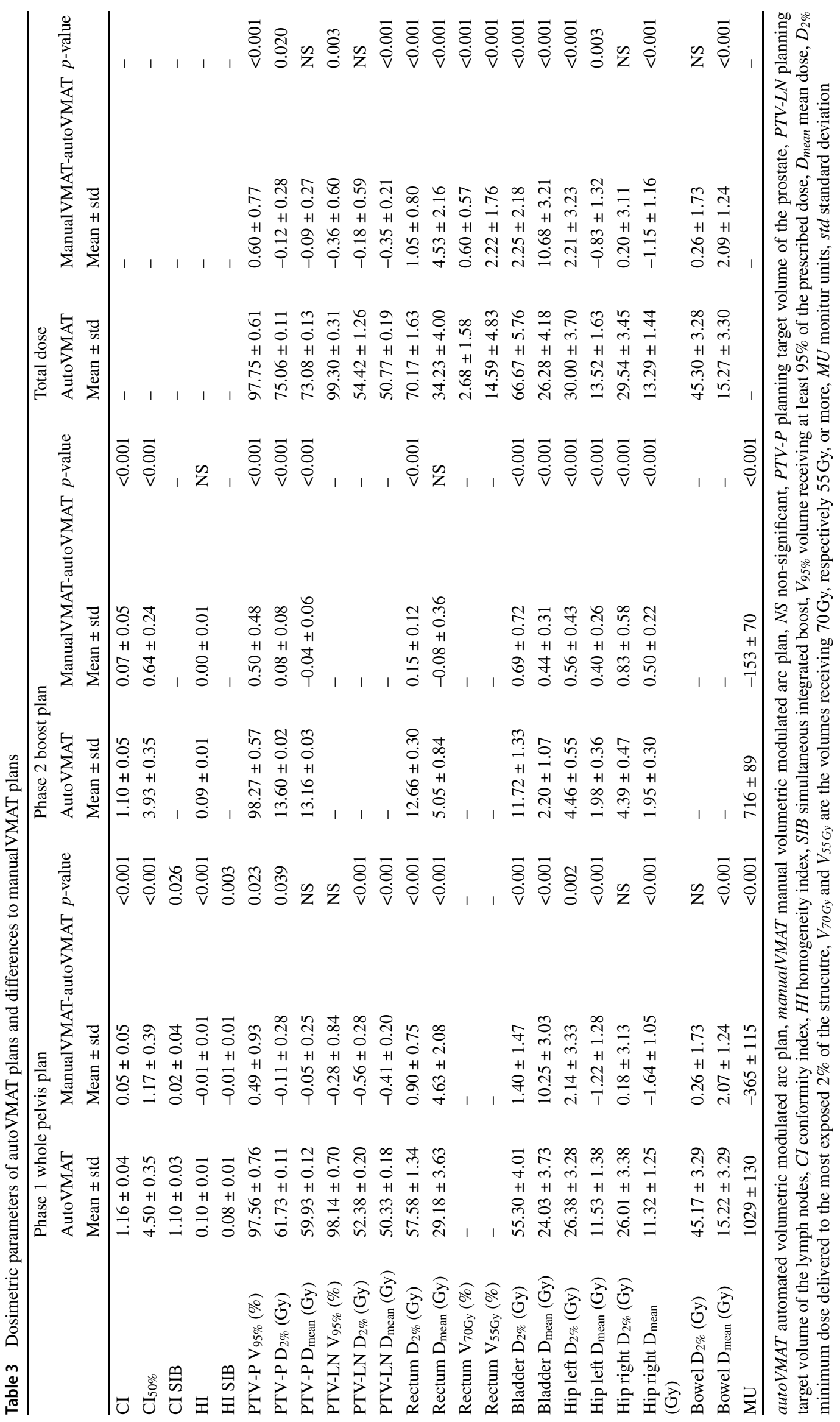



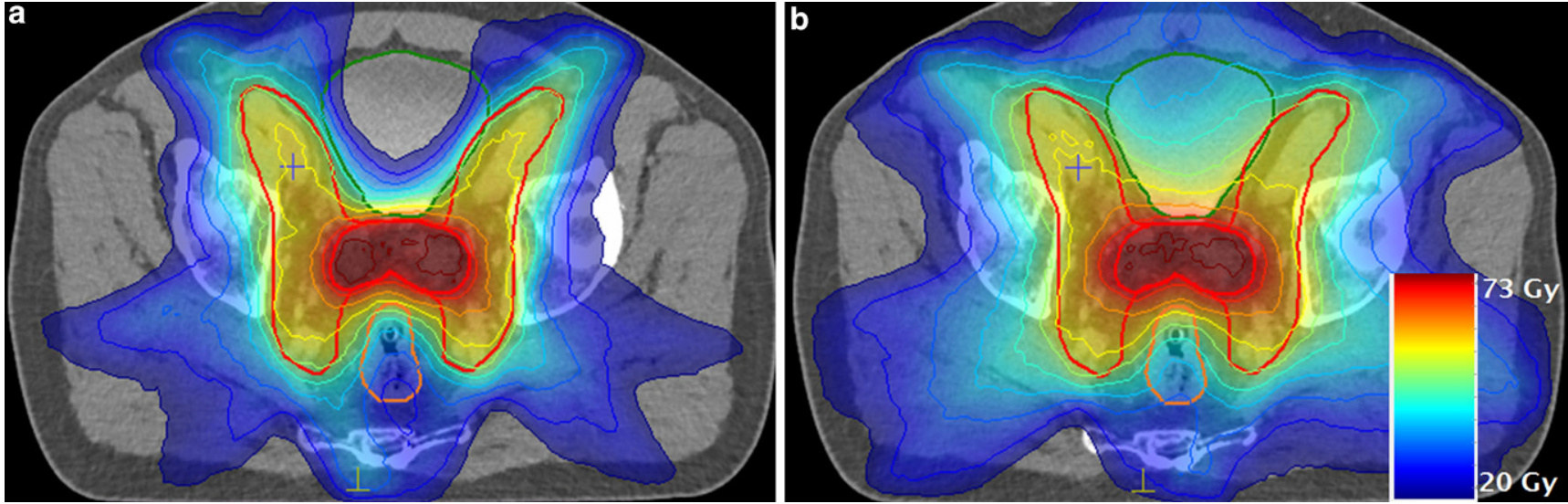

Fig. 2 Exemplary dose distribution of the total summed plan. a Automated volumetric modulated arc plan (autoVMAT); b manual VMAT plan (manualVMAT). Most striking is the improved bladder sparing with autoVMAT, which was also the most important advantage of autoVMAT for the entire study population

held with these 3 patients. The physician then preferred the autoVMAT plans in the second round in all cases.

\section{Planning workload}

The planning workload in manualVMAT was assessed by recording the time for parameter tweaking and reoptimization after the initial optimization run, as the planner would usually not wait in front of the workstation during the first calculation. The average manual planning time was $54 \mathrm{~min}$ for a phase 1 plan and 22 min for a phase 2 plan, which accumulated to $76 \mathrm{~min}$ workload per patient. The additional optimization time in Erasmus-iCycle for autoVMAT was in the range of 3-6h for phase 1 and $20-40$ min for phase 2.

\section{Dosimetric verification measurements}

All treatment plans, both autoVMAT and manualVMAT, exhibited gamma pass rates (GPR) $>95 \%$ in the verification measurements, fulfilling the in-house QA requirements. The mean GPR in phase 1/phase 2 were $98.3 \% / 99.3 \%$ for autoVMAT and $98.6 \% / 99.6 \%$ for manualVMAT. Differences between the verification results of both optimization methods were not statistically significant.

\section{Discussion}

In this study, the potential of fully automated, a priori multicriterial VMAT planning for whole-pelvis prostate treatments was investigated for the first time. This treatment site involves a large concave-shaped target with a SIB, and therefore presents a new and complex optimization problem for the algorithm.

Automated planning significantly improved OAR sparing compared to manual planning. In the total sum plans, the dose to the bladder, rectum, and bowel was substantially reduced. Although a slightly higher target coverage in the prostate PTV was observed in manualVMAT, this small difference was not considered clinically relevant. Additionally, the conformity strongly improved with autoVMAT. The dosimetric advantages were also confirmed by the radiation oncologist, who preferred the autoVMAT plan over manualVMAT in $90 \%$ of cases in a blinded plan comparison session.

The driving forces of autoplanning with Erasmus-iCycle are the wishlists, which essentially define planning protocols or recipes to be followed by the algorithm for automatic plan generation The wishlists are specific for a patient population. In contrast to generally applied planning templates, the wishlists do not need patient-specific fine-tuning. Wishlists are generated in an iterative procedure, using repeated automated plan generations for a small group of test patients.

AutoVMAT planning in this study was indeed fully automatic with no hands-on time, resulting in a reduction of more than 70 min of manual planning time. However, the creation and refining of wishlists in a multidisciplinary team prior to autoplanning for a patient population is time-consuming, which needs to be considered in the departmental logistics.

The number of MUs was significantly higher in autoVMAT than in manualVMAT plans, as a result of higher modulation. The increased plan complexity might lead to challenges in radiation delivery, but no delivery errors could be detected in the verification measurements. More MUs might also lead to more head scatter and higher peripheral doses.

A commercially available a posteriori MCO approach [15] has also demonstrated its potential in WPRT-VMAT planning [16]. Knowledge-based treatment planning strategies have also emerged, which use a model created from 
a library of approved plans to optimize dose distributions for a new patient [17]. However, these approaches still require manual interaction or the establishment of a library of high-quality treatment plans. One limitation of this study is that the Pareto-optimal pre-optimizer (with subsequent reconstruction in Monaco) in autoVMAT was not compared to a manual multi-criterial optimizer $[6,15]$, but rather to current standard clinical practice planning.

Precise concurrent targeting of the prostate and the pelvic lymph nodes with radiation can be challenging, as both target volumes can move independently. The lymph nodes remain relatively fixed to the bony anatomy, while the prostate may shift more than $15 \mathrm{~mm}[18,19]$ in position with variable bladder and rectum filling. Therefore, adaptive RT (ART) has been proposed for WPRT by employing offline replanning or preplanned plan libraries [20-22]. These approaches are rarely clinically realized due to the high workload. With the demonstrated efficiency increase achieved through autoplanning, multiple VMAT plannings and the implementation of ART for WPRT may be feasible.

Another advantage of automated planning is the absence of inter- and intra-planner variations, and higher a homogeneity in plan quality. This may lead to more consistent outcomes in treatment planning studies and clinical trials.

A limitation of the presented (clinical) planning workflow is that the boost plan is created in the initial planning step and possible systematic changes in anatomy over the first weeks of treatment are not incorporated.

Hip prostheses are rather common in the prostate cancer patient population, but these patients were not considered in this study. WPRT planning can be very complex in the presence of hip implants. Voet et al. [23] investigated automated planning for treatment of prostate plus seminal vesicles (no nodes), where automated beam angle optimization was also considered.

Elekta $\mathrm{AB}$ is currently working on the integration of Erasmus-iCycle into their commercial Monaco TPS.

\section{Conclusion}

Fully automated VMAT planning of whole-pelvic prostate treatments with large and complex target volumes created dosimetrically superior treatment plans compared to plans generated manually by experts. The autoVMAT plans were favored by the radiation oncologist in $90 \%$ of patients and the planning workload was considerably reduced.

Acknowledgements The authors thank Alireza Homayuni for his support. The financial support from the Austrian Federal Ministry of Science, Research and Economy and the National Foundation for Research, Technology and Development is gratefully acknowledged.
Funding Open access funding provided by Medical University of Vienna.

\section{Compliance with ethical guidelines}

Conflict of interest The department of Radiation Oncology of Erasmus MC Cancer Institute has research collaborations with Elekta AB, Stockholm, Sweden and Accuray Inc, Sunnyvale, USA. M. Buschmann, A.W.M. Sharfo, J. Penninkhof, Y. Seppenwoolde, G. Goldner, D. Georg, S. Breedveld, and B.J. M. Heijmen declare that they have no competing interests.

Ethical standards This retrospective analysis does not contain any studies with human participants or animals performed by any of the authors. Informed consent from individual participants was not needed. The study was approved by the institutional ethics committee.

Open Access This article is distributed under the terms of the Creative Commons Attribution 4.0 International License (http:// creativecommons.org/licenses/by/4.0/), which permits unrestricted use, distribution, and reproduction in any medium, provided you give appropriate credit to the original author(s) and the source, provide a link to the Creative Commons license, and indicate if changes were made.

\section{References}

1. Roach M, DeSilvio M, Lawton C, Uhl V, Machtay M, Seider MJ et al (2003) Phase III trial comparing whole-pelvic versus prostateonly radiotherapy and neoadjuvant versus adjuvant combined androgen suppression: Radiation Therapy Oncology Group 9413. J Clin Oncol 21:1904-1911

2. Mantini G, Tagliaferri L, Mattiucci GC, Balducci M, Frascino V, Dinapoli N et al (2011) Effect of whole pelvic radiotherapy for patients with locally advanced prostate cancer treated with radiotherapy and long-term androgen deprivation therapy. Int J Radiat Oncol Biol Phys 81:e721-e726

3. Amini A, Jones BL, Yeh N, Rusthoven CG, Armstrong H, Kavanagh BD (2015) Survival outcomes of whole-pelvic versus prostate-only radiation therapy for high-risk prostate cancer patients with use of the National Cancer Data Base. Int J Radiat Oncol Biol Phys 93:1052-1063

4. Morikawa LK, Roach M (2011) Pelvic nodal radiotherapy in patients with unfavorable intermediate and high-risk prostate cancer: evidence, rationale, and future directions. Int J Radiat Oncol Biol Phys 80:6-16

5. Craft D, Halabi T, Shih HA, Bortfeld T (2007) An approach for practical multiobjective IMRT treatment planning. Int J Radiat Oncol Biol Phys 69:1600-1607

6. Craft D, Monz M (2010) Simultaneous navigation of multiple Pareto surfaces, with an application to multicriteria IMRT planning with multiple beam angle configurations. Med Phys 37:736-741

7. Breedveld S, Storchi PRM, Voet PWJ, Heijmen BJM (2012) iCycle: Integrated, multicriterial beam angle, and profile optimization for generation of coplanar and noncoplanar IMRT plans. Med Phys 39:951-963

8. Voet PWJ, Dirkx MLP, Breedveld S, Fransen D, Levendag PC, Heijmen BJM (2013) Toward fully automated multicriterial plan generation: a prospective clinical study. Int J Radiat Oncol Biol Phys 85:866-872

9. Voet PWJ, Dirkx MLP, Breedveld S, Al-Mamgani A, Incrocci L, Heijmen BJM (2014) Fully automated volumetric modulated arc therapy plan generation for prostate cancer patients. Int J Radiat Oncol Biol Phys 88:1175-1179 
10. Gala DG, Dirkx MLP, Hoekstra N, Fransen D, Lanconelli N, van de Pol M et al (2017) Fully automated VMAT treatment planning for advanced-stage NSCLC patients. Strahlenther Onkol 193:402-409

11. Sharfo AWM, Voet PWJ, Breedveld S, Mens JWM, Hoogeman MS, Heijmen BJM (2015) Comparison of VMAT and IMRT strategies for cervical cancer patients using automated planning. Radiother Oncol 114:395-401

12. Sharfo AWM, Breedveld S, Voet PWJ, Heijkoop ST, Mens J-WM, Hoogeman MS et al (2016) Validation of fully automated VMAT plan generation for library-based plan-of-the-day cervical cancer radiotherapy. PLoS ONE 11:e169202. https://doi.org/10.1371/ journal.pone. 0169202

13. Buergy D, Sharfo AWM, Heijmen BJM, Voet PWJ, Breedveld S, Wenz F et al (2017) Fully automated treatment planning of spinal metastases - A comparison to manual planning of volumetric modulated arc therapy for conventionally fractionated irradiation. Radiat Oncol 12:33

14. Michalski JM, Gay H, Jackson A, Tucker SL, Deasy JO (2010) Radiation dose-volume effects in radiation-induced rectal injury. Int J Radiat Oncol Biol Phys 76:S123-9

15. Bokrantz R (2012) Multicriteria optimization for volumetric-modulated arc therapy by decomposition into a fluence-based relaxation and a segment weight-based restriction. Med Phys 39:6712-6725

16. Buschmann M, Seppenwoolde Y, Wiezorek T, Weibert K, Georg D (2016) Advanced optimization methods for whole pelvic and local prostate external beam therapy. Phys Med 32:465-473
17. Hussein M, South CP, Barry MA, Adams EJ, Jordan TJ, Stewart AJ et al (2016) Clinical validation and benchmarking of knowledgebased IMRT and VMAT treatment planning in pelvic anatomy. Radiother Oncol 120:473-479

18. Peng C, Ahunbay E, Chen G, Anderson S, Lawton C, Li XA (2011) Characterizing interfraction variations and their dosimetric effects in prostate cancer radiotherapy. Int J Radiat Oncol Biol Phys 79:909-914

19. Bylund KC, Bayouth JE, Smith MC, Hass AC, Bhatia SK, Buatti JM (2008) Analysis of interfraction prostate motion using megavoltage cone beam computed tomography. Int J Radiat Oncol Biol Phys 72:949-956

20. Qi P, Pouliot J, Roach M, Xia P (2014) Offline multiple adaptive planning strategy for concurrent irradiation of the prostate and pelvic lymph nodes. Med Phys 41:21704

21. Cantin A, Gingras L, Lachance B, Foster W, Goudreault J, Archambault L (2015) Dosimetric evaluation of three adaptive strategies for prostate cancer treatment including pelvic lymph nodes irradiation. Med Phys 42:7011-7021

22. McVicar N, Popescu IA, Heath E (2016) Techniques for adaptive prostate radiotherapy. Phys Med 32:492-498

23. Voet PWJ, Dirkx MLP, Breedveld S, Heijmen BJM (2013) Automated generation of IMRT treatment plans for prostate cancer patients with metal hip prostheses: comparison of different planning strategies. Med Phys 40:71704 